\title{
Posttraumatic triggering of the extensor pollicis brevis tendon in de Quervain's disease successfully diagnosed with ultrasonography: A case report
}

Takuya Uemura, Koichi Yano, Yusuke Miyashima, Sadahiko Konishi, Hiroaki Nakamura

\begin{tabular}{|c|c|}
\hline Citation & Journal of Clinical Ultrasound. 49(4); 398-400 \\
\hline Issue Date & $2021-05$ \\
\hline Version of Record & $2020-10-06$ \\
\hline Type & Journal Article \\
\hline Textversion & Author \\
\hline $\begin{array}{l}\text { Supporting } \\
\text { Information }\end{array}$ & $\begin{array}{l}\text { Supporting Information is available at https://doi.org/10.1002/jcu.22934. } \\
\text { Video S1: Videoclip 1. Preoperative videorecording shows the snapping at the right } \\
\text { radial wrist. } \\
\text { Video S2: Videoclip 2. Pre- and postoperative dynamic sonographic examinations. } \\
\text { Video S3: Videoclip 3. Intraoperative videorecording during the wide-awake surgery } \\
\text { documents the triggering of the extensor pollicis brevis tendon and then the release } \\
\text { of the extensor pollicis brevis subcompartment. }\end{array}$ \\
\hline Rights & $\begin{array}{l}\text { This is the peer reviewed version of the following article: Journal of Clinical Ultrasound. } \\
\text { Volume49, Issue4, Pages } 398-400 \text {., which has been published in final form at } \\
\text { https://doi.org/10.1002/jcu.22934. This article may not be enhanced, enriched or } \\
\text { otherwise transformed into a derivative work, without express permission from Wiley or } \\
\text { by statutory rights under applicable legislation. Copyright notices must not be removed, } \\
\text { obscured or modified. The article must be linked to Wiley's version of record on Wiley } \\
\text { Online Library and any embedding, framing or otherwise making available the article or } \\
\text { pages thereof by third parties from platforms, services and websites other than Wiley } \\
\text { Online Library must be prohibited. }\end{array}$ \\
\hline DOI & 10.1002/jcu.22934 \\
\hline
\end{tabular}

Self-Archiving by Author(s)

Placed on: Osaka City University Repository

Uemura, T., Yano, K., Miyashima, Y., Konishi, S., \& Nakamura, H. (2020). Posttraumatic triggering of the extensor pollicis brevis tendon in de Quervain's disease successfully diagnosed with ultrasonography: A case report. Journal of Clinical Ultrasound, 49(4), 398-400. https://doi.org/10.1002/jcu.22934 
Posttraumatic triggering of the extensor pollicis brevis tendon in de Quervain's disease successfully diagnosed with ultrasonography: A case report

Takuya Uemura M.D., Ph.D. ${ }^{1,3,}{ }^{*}$, Koichi Yano M.D., Ph.D. ${ }^{2}$, Yusuke Miyashima M.D. ${ }^{1}$, Sadahiko Konishi M.D., Ph.D. ${ }^{1}$, Hiroaki Nakamura M.D., Ph.D. ${ }^{3}$

${ }^{1}$ Department of Orthopaedic Surgery, Osaka General Hospital of West Japan Railway

Company, Osaka, Japan.

${ }^{2}$ Department of Orthopaedic Surgery, Seikeikai Hospital, Osaka, Japan.

${ }^{3}$ Department of Orthopaedic Surgery, Osaka City University Graduate School of Medicine, Osaka, Japan.

*Corresponding author: Takuya Uemura, M.D., Ph.D., Department of Orthopaedic

Surgery, Osaka General Hospital of West Japan Railway Company, 1-2-22

Matsuzakicho, Abeno-ku, Osaka 545-0053, Japan. Tel: +81-6-6628-2221. Fax:

+81-6-6628-4707. E-mail: t-uemura@med.osaka-cu.ac.jp 
Running title

Triggering in de Quervain's disease diagnosed with ultrasonography

\section{Conflict of Interest}

The authors declare that they have no conflict of interest.

Acknowledgments

We would like to thank Editage (www.editage.com) for English language editing. 


\begin{abstract}
Triggering due to de Quervain's disease is exceedingly rare. This is the first successfully diagnosed case of the snapping phenomenon of the extensor pollicis brevis (EPB) tendon in de Quervain's disease evaluated with preoperative dynamic ultrasonography, clearly demonstrated in an intraoperative video, and treated with decompression of EPB subcompartment only under a wide-awake surgery. Dynamic ultrasonographic images identified snapping caused by unsmooth excursion of an enlarged EPB tendon in a separate subcompartment. In snapping de Quervain's disease, ultrasonographic evaluations and wide-awake surgery are essential to exactly diagnose and successfully treat the snapping condition of the EPB and/or APL tendons.
\end{abstract}

Key words: trigger thumb, entrapment, abductor pollicis longs, first dorsal extensor compartment, wide awake surgery

\title{
Introduction
}

Extensor triggering due to de Quervain's disease, showing a pseudotriggering of the 
thumb, is exceedingly rare with only a few previous reports in the English literature. ${ }^{1-3}$

This is the first successfully diagnosed posttraumatic case of the snapping phenomenon of the extensor pollicis brevis (EPB) tendon in de Quervain's disease evaluated with preoperative dynamic ultrasonography, clearly demonstrated in intraoperative procedure recorded in a video and treated with EPB subcompartment decompression only under a wide-awake surgery.

\section{Case Report}

A 52-year-old man complained of painful snapping at the right radial wrist on attempted extension of his thumb for 2 weeks since falling and injuring himself during a karate session. On physical examination, tenderness and palpable snapping were observed over the radial styloid process, during active extension and flexion of the metacarpophalangeal joint of the patient's thumb (Video 1). Eichhoff test result was positive. Ultrasonography using SONIMAGE HS1 (KONICA MINOLTA, INC., Japan) with a L18-4 ultra-broadband linear transducer (10 MHz) showed a swollen EPB tendon in a separate subcompartment with an enlarged vertical septum in the first extensor 
compartment, as compared with the unaffected left side (Figure 1). The tendon sheath of the EPB subcompartment also became thick, whereas the tendon sheath of the abductor pollicis longus (APL) remained normal. Moreover, dynamic ultrasonographic images clearly identified a snapping phenomenon caused by unsmooth excursion of an enlarged EPB tendon at the proximal portion of the first extensor compartment (Video 2). Based on these findings, the patient was diagnosed with posttraumatic de Quervain's disease accompanied with EPB tendon triggering. Two doses of triamcinolone injected into the EPB subcompartment were temporarily effective for pain relief; however, painful snapping still recurred. Finally, as the intense pain (visual analogue scale [VAS] score of $78 \mathrm{~mm}$ ) and thumb motion impairment with snapping persisted, surgical intervention was performed 16 months after the onset of symptoms.

Wide-awake surgery under local anesthesia with epinephrine was chosen to reproduce the snapping phenomenon under a voluntary thumb motion. A 4-cm longitudinal skin incision was made along the EPB tendon over the radial styloid process. After the identification and gentle dorsal retraction of the superficial branch of the radial nerve, the EPB tendon and its subcompartment sheath were detected. 
Intraoperatively, triggering of the EPB tendon was observed at the proximal part of the

first extensor compartment, such as preoperative dynamic ultrasonography images,

while the patient actively extended or flexed the metacarpophalangeal joint of his thumb

(Video 3). Triggering and entrapment of the APL tendons including some accessory

slips was not observed. Therefore, only the tendon sheath of the EPB was released. In a complete separate fibro-osseous tunnel of the first extensor compartment, an enlarged

EPB tendon with partially discolored hourglass-shaped nodule was detected (Figure 2).

Snapping of the EPB tendon was caused by localized swelling of the EPB tendon that

was triggered within the narrowed fibro-osseous tunnel of the EPB subcompartment.

After decompressing only the EPB subcompartment, we confirmed the disappearance of snapping under active intraoperative movements of his thumb. To prevent the released EPB tendon from volar subluxation, the tendon sheath was loosely reconstructed with the retinacular sling flap using the dorsal end of the remnant sheath. ${ }^{4}$ Finally, snapping and entrapment of the EPB tendon were resolved without tendon subluxation during the active thumb and wrist movements. 
Postoperatively, a splint was used with the gently extended wrist and thumb for 2 weeks. At 6 months postoperatively, patients had complete pain relief (VAS $0 \mathrm{~mm}$ ); there was no snapping or tenderness over the first extensor compartment. Eichhoff test result was negative. Grip strength for the right hand increased from $28 \mathrm{~kg}$ preoperatively to $36 \mathrm{~kg}$ postoperatively. Patient-reported outcomes measured by the Quick Disability of the Arm, Shoulder, and Hand and the Hand 20 were improved from 81.8 and 80 preoperatively to 9.1 and 3.5 postoperatively, respectively. The patient provided written informed consent to publish the cases. The patient was informed that data from the case would be submitted for publication, and gave their consent, and this study has been approved by the institutional review board of the authors' affiliated institutions.

\section{Discussion}

Triggering is an uncommon symptom in de Quervain's disease with a prevalence of only $1.3 \%$ based on a review of 827 consecutive patients over a 5 -year period as previously reported. ${ }^{3}$ Triggering due to de Quervain's disease was first reported in a 
child in 1979, which probably should not be considered a true de Quervain's tenosynovitis, and later reported in several small case series that comprise a total of 14 operative cases. ${ }^{1-6}$ Among them, a posttraumatic case of snapping de Quervain's disease like that in the present case was exceedingly rare with only 1 case reported in the literature. ${ }^{3}$ Ultrasound has been used for the assessment of de Quervain's disease and has appeared as an useful tool in the investigation of triggering or snapping phenomena in various regions of the body. ${ }^{7,8}$ However, neither pre- and postoperative dynamic ultrasonographic images nor intraoperative video has demonstrated the snapping de Quervain's disease.

No unique anatomic cause of triggering in de Quervain's disease was identified. As previously reported, triggering of the first dorsal compartment may result from nodularity in the EPB, APL, or both tendons in association with stenosis and synovitis in the fibroosseous tunnel. ${ }^{1-4}$ In this posttraumatic case, a discolored hourglass-shaped nodule in the EPB tendon was suggested to facilitate the intratendinous healing process after a traumatic direct and/or indirect tendon damage.

With regard to operative treatment for snapping de Quervain's disease, determining 
which subcompartment should be released remains controversial. Some authors

advocated releasing the subcompartment only for the EPB tendon. ${ }^{1,9}$ Another

recommended release was both subcompartments for EPB and APL tendons because

they might have nodularity. ${ }^{2-4,10}$ To resolve this problem, preoperative ultrasonographic

evaluations help identify the nodularity in the EPB and/or APL tendons with or without

tenosynovitis and decide the release of subcompartment. Moreover, the wide-awake

local anesthesia no tourniquet (WALANT) surgical technique is also useful to determine

the snapping subcompartment and confirm disappearance of snapping after the release

under voluntary movements of the patient's thumb intraoperatively. ${ }^{11}$

In triggering due to de Quervain's disease, preoperative ultrasonographic evaluations and WALANT surgery are essential to exactly diagnose and successfully treat the snapping condition of the EPB and/or APL tendons with nodularity in each subcompartment.

\section{References}

1. Viegas SF. Trigger thumb of de Quervain's disease. J Hand Surg Am. 
$1986 ; 11: 235-237$.

2. Witczak JW, Masear VR, Meyer RD. Triggering of the thumb with de

Quervain's stenosing tendovaginitis. J Hand Surg Am. 1990;15:265-268.

3. Alberton GM, High WA, Shin AY, et al. Extensor triggering in de Quervain's stenosing tenosynovitis. J Hand Surg Am. 1999;24:1311-1314.

4. Greenhill DA, Thoder JJ, Abdelfattah H. Triggering of the abductor pollicis longus in association with deQuervain's tenosynovitis. BMJ Case Rep. $2017 ; 2017$.

5. Chow SP. Triggering due to de Quervain's disease. Hand. 1979;11:93-94.

6. Chang KV, Hung CY, Özçakar L. Snapping Thumb and Superficial Radial Nerve Entrapment in De Quervain Disease: Ultrasound Imaging/Guidance Revisited. Pain Med. 2015;16:2214-2215.

7. McBain B, Rio E, Cook J, et al. Diagnostic accuracy of imaging modalities in the detection of clinically diagnosed de Quervain's syndrome: a systematic review. Skeletal Radiol. 2019;48:1715-1721.

8. Guillin R, Marchand AJ, Roux A, et al. Imaging of snapping phenomena. Br J 
Radiol. 2012;85:1343-1353.

9. Yuasa K, Kiyoshige Y. Limited surgical treatment of de Quervain's disease:

decompression of only the extensor pollicis brevis subcompartment. J Hand Surg Am. 1998;23:840-843.

10. Maruyama M, Takahara M, Kikuchi N, et al. De Quervain disease caused by abductor pollicis longus tenosynovitis: a report of three cases. Hand Surg. $2009 ; 14: 43-47$.

11. Lalonde DH. Conceptual origins, current practice, and views of wide awake hand surgery. J Hand Surg Eur Vol. 2017;42:886-895. 


\section{Figure Legends}

Figure 1. Preoperative ultrasonographic images in the axial (A) and sagittal (B) planes

show the localized swelling (arrow) of the extensor pollicis brevis tendon (asterisk) in a separate subcompartment with an enlarged vertical septum (white circle) in the right first extensor compartment, as compared with the unaffected left side. The tendon sheath of the abductor pollicis longus (white triangle) does not become thick. 
Figure 1
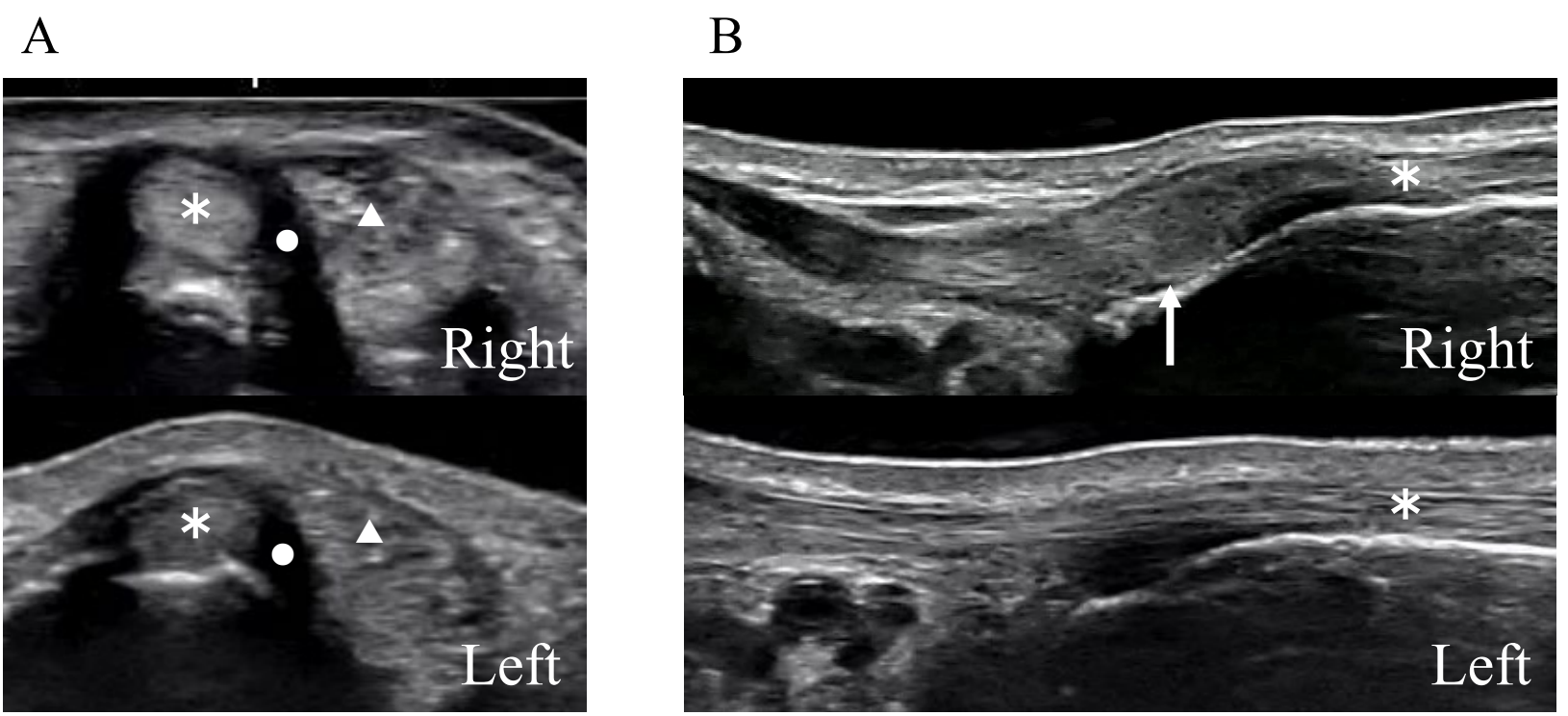
Figure 2. A. Intraoperative findings show the partially enlarged extensor pollicis brevis tendon (asterisk) with an hourglass-shaped nodule (arrow). B. A complete separate fibro-osseous tunnel of the extensor pollicis brevis (asterisk) subcompartment was released and the subcompartment of the abductor pollicis longus tendon (black triangle) was not released. 
Figure 2

A

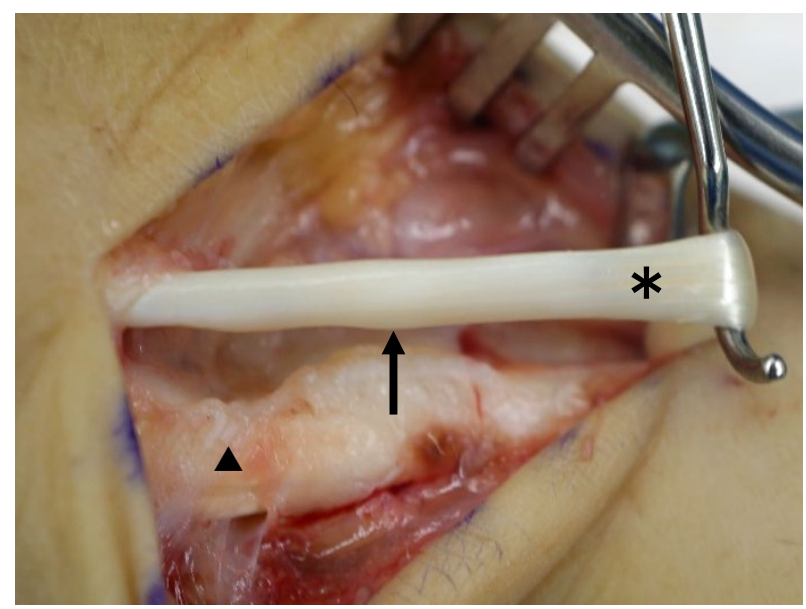

B

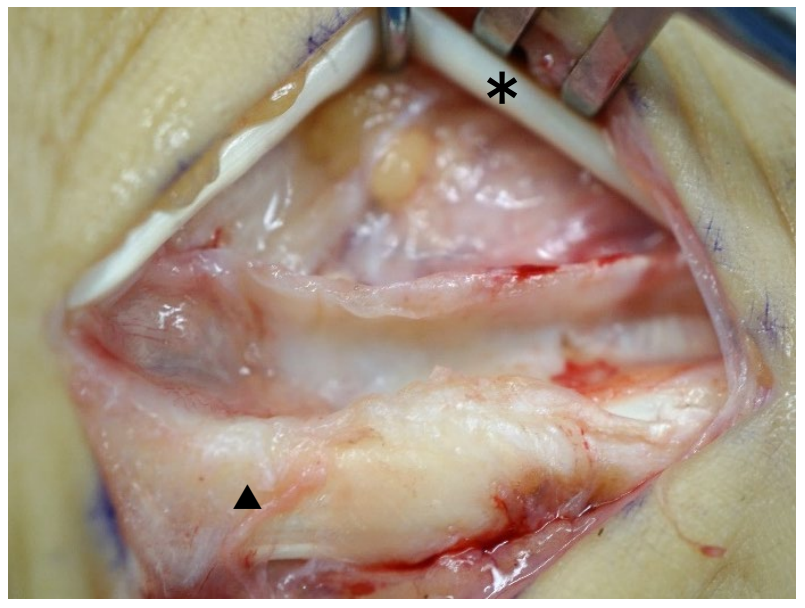




\section{Video Legends}

Video 1. Preoperative video of snapping at the right radial wrist.

Video 2. Pre- and postoperative dynamic ultrasonographic images.

Video 3. Intraoperative video of triggering of the extensor pollicis brevis tendon and releasing of the extensor pollicis brevis subcompartment. 